\title{
Vascular Stenosis Asymmetry Influences Considerably Pressure Gradient and Flow Volume
}

\author{
L. NOVAKOVA ${ }^{1}$, J. KOLINSKY $^{1}$, J. ADAMEC $^{1}$, J. KUDLICKA ${ }^{2}$, J. MALIK $^{2}$ \\ ${ }^{1}$ Department of Fluid Mechanics and Thermomechanics, Faculty of Mechanical Engineering, Czech \\ Technical University in Prague, Czech Republic, ${ }^{2}$ Third Department of Internal Medicine, First \\ Medical Faculty, Charles University in Prague, Czech Republic
}

Received October 9, 2014

Accepted August 28, 2015

On-line November 24, 2015

\begin{abstract}
Summary
Vascular stenosis is often described only by its percentage in both clinical and scientific praxis. Previous studies gave inconclusive results regarding the effect of stenosis eccentricity on its hemodynamic effect. The aim of this experimental study was to investigate and quantify the effect of stenosis severity and eccentricity on the pressure drop. A combination of pressure and flow measurements by Particle Imaging Velocimetry (PIV) method was used. Models of the same stenosis significance but with different levels of eccentricity were studied in vitro by PIV. This study has shown that stenosis asymmetry is associated with more profound pressure drop and flow volume decrease. On the contrary, pressure drop and flow volume decrease were not further significantly influenced by the level of asymmetry. Hemodynamic changes associated with stenosis eccentricity must be taken into account in both clinical and scientific studies.
\end{abstract}

\section{Key words}

Stenosis • Atherosclerosis • Dialysis access • Pressure gradient

\section{Corresponding author}

L. Novakova, Department of Fluid Dynamics and Thermodynamics, Faculty of Mechanical Engineering, Czech Technical University in Prague, Technicka 4, 16607 Prague, Czech Republic. E-mail: Ludmila.novakova@fs.cvut.cz

\section{Introduction}

Stenosis is undoubtedly the most frequent vascular complication. The main etiology is atherosclerosis, but stenosis can develop also as a result of medial calcinosis in patients with diabetes mellitus, by vasculitis, outer pressure on the artery etc. Clinically, stenoses manifest by the ischemia of the particular part of the body or organ, such as coronary heart disease, intermittent claudication, cerebral stroke and others. Various physiological mechanisms in the human body influence the development of ischemia, such as the variations in the arterial blood pressure and in the peripheral vascular resistance. The clinical picture depends on the time of stenosis development (acute vs. chronic) and on the hemodynamic significance of the stenosis. The latter is, however, often a source of misunderstandings. The problem is that various aspects and imaging methods are applied in the stenosis assessment. Clinically, a stenosis is significant, when it causes ischemia symptoms. Imaging methods include nowadays angiography, duplex Doppler ultrasonography, computerized tomography or magnetic resonance angiography, but also more functional approach is used, such as measurement of the fractional flow reserve (Groves et al. 2014) and/or of the pressure gradient (Wilmink 2014). Stenosis severity is usually described as the percentage area reduction relative to the area of nonconstricted vessel. Imaging methods consider generally a stenosis significant, when it is $\geq 75 \%$. Atherosclerotic plaques develop sooner in regions with disturbed, low wall shear stress, such as in the outer walls of bifurcations, inner arch of the aorta and others (Malik et al. 2012). As a result, many stenoses are asymmetric, which changes the flow characteristics. However, this fact is frequently neglected in both clinical praxis and 
experimental work, where angiography is used as the golden standard method. Various mechanisms involved in the stenosis hemodynamic consequences are studied in detail by the fluid dynamics.

From the fluid dynamics point of view stenosis creates a pressure drop and represents the obstruction that disturbs the flow. Characteristic flow field pattern can be observed behind the stenosis. Flow accelerates in the converging part of the stenosis and the jet is formed at the throat of the stenosis. The diameter of the jet could be even smaller than the inner diameter of the stenosis. It is caused by the effect of inertial forces acting on the liquid while changing the direction of trajectory. The velocity profile of the jet expands gradually and smoothly downstream of the stenosis. The flow in the axisymmetric stenosis model experiences an axisymmetric recirculation zone in peripheral regions. This zone is driven by the high velocity jet formed in the valve and is characterized by higher energy dissipation. All these effects influence the final pressure loss. In vitro experimental studies of flow field in vessel narrowing with a variable degree of lumen reduction are generally focused on models with a solid (Liu 2007, Shuib et al. 2011) as well as elastic wall (Pielhop et al. 2011). The fluid flow patterns and wall shear stress in both axisymmetric and asymmetric stenosis have been examined in studies of Heflin et al. (2009) and Tang et al. (2013). Apart from numerical studies, there are only a few comparative studies on pressure drop (Lundin et al. 2003, Roy, 2006, Young et al. 1972).

The aim of this experimental study was to investigate and quantify the effect of stenosis severity and eccentricity on the pressure drop. A combination of pressure and flow measurements by Particle Imaging Velocimetry (PIV) method was used. This combination allowed us to relate the geometry of the stenosis and the pressure loss.

\section{Methods}

In this study we experimentally investigated the loss coefficient of various stenotic models. Our stenosis models were made of $39 \mathrm{~mm}$ long acrylic tube with the diameter of $26 \mathrm{~mm}$, the range of area reductions was between $25 \%$ and $75 \%$. The reduced diameter was calculated as

$$
d_{2}=d_{1} \sqrt{ }\left(1-\frac{c}{100}\right)
$$

where $\mathrm{c}$ is the percentage of reduction (\%). Seven various geometries of stenosed pipes were measured. These included: symmetrical concentric constrictions with $25 \%, 50 \%$ or $75 \%$ reduction of active cross section, $50 \%$ and $75 \%$ eccentric constrictions with two different degrees of eccentricity. Eccentricity of the stenosis was determined as

$$
\mathrm{e}=\frac{z}{r_{2}-r_{1}} \cdot 100
$$

where $r_{2}$ is the inner radius of the unstenosed tube $\left(r_{2}=d_{2} / 2\right)$ and $r_{1}$ is the inner radius of the stenosis $\left(r_{1}=d_{1} / 2\right)$. To simplify the stenosis model and to get comparable results, we used constant stenosis total length. The square-ended $75 \%$ stenosis with sudden contraction and expansion was measured as well for comparison. The geometry of all stenoses and dimensions are stated in Figure 1.

For steady case, the law of similarity is governed by Reynolds number, therefore we equalized the Reynolds numbers between the real and modeled stenosis. Sets of experiments were carried out with Reynolds number from 100 to 3000 , corresponding with flow rate from $120 \mathrm{ml} / \mathrm{min}$ to $3700 \mathrm{ml} / \mathrm{min}$. The characteristic parameters were the average flow velocity ( $v$ mean) and the hydraulic diameter $\left(d_{2}\right)$.

The pressure loss vs. flow dependency was measured in vitro. The flow rate was controlled by a pump capable of supplying a steady flow with adjustable flow rate. A specially designed connector was used for fitting the semiconductor pressure transducer for gauge pressure measurement. The static pressure was measured $15 \mathrm{~cm}$ in front of and behind of the stenosis. The pressure transducer outputs were sampled at $1 \mathrm{kHz}$ with an analogue/digital converter and the data were filtered to reduce noise and random fluctuations. The transducers were calibrated using U-manometer. Before each experiment the pressure transducers were zeroed and the pump programmed to generate an accurate flow rate.

In fluid dynamics the relation for pressure drop $\Delta p$ in a pipe with stenosis is assumed in the form (Idelchik 2005):

$$
\Delta \mathrm{p}=\Delta \mathrm{p}_{\text {friction }}+\Delta \mathrm{p}_{\text {local loss }}
$$

The first term introduces the pressure drop due to the friction in the narrow parts of the tube. This pressure loss increases linearly with velocity for laminar flow regime; the latter term involves the pressure local loss associated with the changes in cross-section within the stenosis. The pressure local loss and velocity has 
a second power relation between them. In case of stenosis the considered length is small and therefore friction losses can be neglected with acceptable error. This simplification is valid in the range of Reynolds number stated in table in Figure 1. For lower Reynolds number the effect of friction losses cannot be neglected. Considering only local loss of stenosis (Idelchik 2005):

$$
\Delta \mathrm{p}=\xi \frac{\mathrm{v}^{2}}{2} \rho
$$

where $\mathrm{v}$ is the mean velocity in the narrow part of the tube. Using Equation 2 the pressure loss is related to some particular dynamic pressure by the dimensionless loss coefficient $\zeta$. Neglecting friction, this coefficient is considered to be constant in our study. The value of this coefficient ranges from 0 upwards and should be determined experimentally. For $\zeta=1$ the pressure drop is equal to the dynamic pressure. The pressure coefficient of each stenosis was computed from measured data after the friction loss of narrow tube with identical length were subtracted. The knowledge of the loss coefficient allows us to assess the effect of stenosis and to compare various types of stenoses. The decrease of flow volume was expressed by the ratio $Q / Q_{\max }$ (which is physiologically valid if the perfusion pressure and peripheral vascular resistance remain stable). $Q_{\max }$ is the maximal flow rate which can occur for stenosis with $0 \%$ of area reduction. $Q$ is the actual flow rate for given degree of stenosis. For the ratio $Q / Q_{\max }$ the modified Bernoulli equation expressed in terms of volume flow can be applied (Idelchik 2005):

$$
\frac{\mathrm{Q}}{\mathrm{Q}_{\max }}=\frac{1}{\sqrt{1+\xi}}
$$

This comparative parameter that involves the pressure loss coefficient gives the information about functional severity of stenosis.

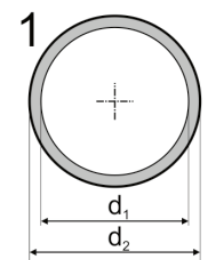

$25 \%$

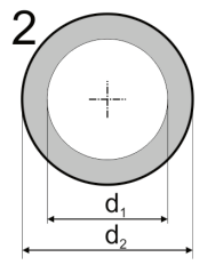

$50 \%$

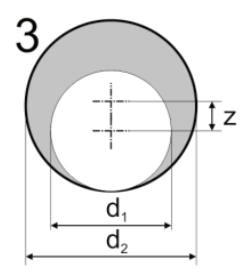

$50 \%$

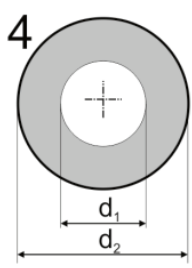

$75 \%$

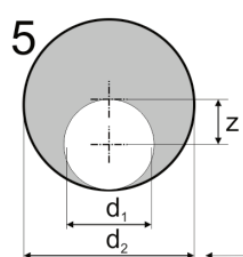

$75 \%$

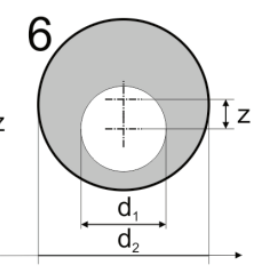

$75 \%$

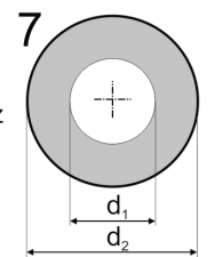

$75 \%$

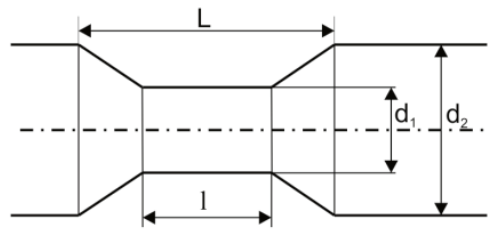

concentric

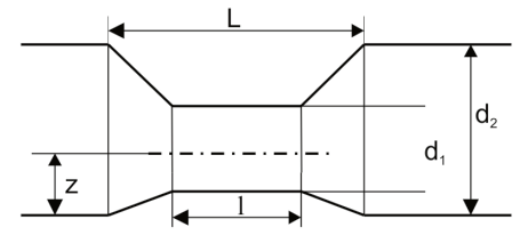

eccentric

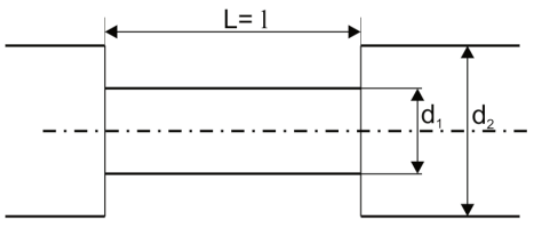

\begin{tabular}{|c|c|c|c|c|c|c|c|c|c|}
\hline & Stenosis model & \begin{tabular}{|l} 
Tube \\
diameter \\
$d_{2}(\mathrm{~mm})$ \\
\end{tabular} & $\begin{array}{l}\text { Neck } \\
\text { diameter } \\
d_{1}(\mathrm{~mm}) \\
\end{array}$ & \begin{tabular}{|l} 
Length of \\
model L \\
$(\mathrm{mm})$
\end{tabular} & \begin{tabular}{|l|} 
Length of \\
constriction \\
I $(\mathrm{mm})$
\end{tabular} & $\begin{array}{l}\text { Distance } \\
\text { from axis } \\
\mathrm{z}(\mathrm{mm}) \\
\end{array}$ & \begin{tabular}{|l|} 
Eccentr \\
icity e \\
$(\%)$ \\
\end{tabular} & $\begin{array}{l}\text { Pressure loss } \\
\text { coefficient } \\
(1)\end{array}$ & $\begin{array}{l}\text { R- } \\
\text { square } \\
\text { d } \\
\end{array}$ \\
\hline 1 & Concentric $25 \%$ & 26 & 22.5 & 39 & 33.7 & 0 & 0 & $0.17(\operatorname{Re}>2000)$ & 0.97 \\
\hline 2 & Concentric $50 \%$ & 26 & 18.4 & 39 & 27.6 & 0 & 0 & $1.1(\operatorname{Re}>1000)$ & 0.99 \\
\hline 3 & Eccentric $50 \%$ & 26 & 18.4 & 39 & 27.6 & 3.8 & 100 & $2.15(\operatorname{Re}>1000)$ & 0.99 \\
\hline 4 & Concentric $75 \%$ & 26 & 13 & 39 & 19.5 & 0 & 0 & $10.7(\operatorname{Re}>500)$ & 0.99 \\
\hline 5 & Eccentric I. 75 \% & 26 & 13 & 39 & 19.5 & 6.5 & 100 & $14.9(\operatorname{Re}>500)$ & 0.99 \\
\hline 6 & Eccentric II. 75\% & 26 & 13 & 39 & 19.5 & 3.8 & 58 & $14.5(\operatorname{Re}>500)$ & 0.99 \\
\hline 7 & $\begin{array}{l}\text { Sudden } \\
\text { contraction } 75 \%\end{array}$ & 26 & 13 & 39 & 39 & 0 & 0 & 15.3 & 0.99 \\
\hline
\end{tabular}

sudden contraction

Fig. 1. Schematic diagram of the cross-sections of all types of idealized machined, rigid, stenosis used in the in vitro experiments: $1,2,4$ concentric stenosis; 3,5,6 eccentric stenosis with various degree of contraction and eccentricity; 7-concentric stenosis with sudden contraction. 


\section{Results}

Table in Figure 1 summarizes the complete set of experimental results for all measured stenoses. The steady flow resistance was found to vary quadratically with flow (coefficients of determination of polynomial regression R-squared are referred in table in Figure 1) confirming the validity of Equation 2. The loss coefficient of the concentric stenosis was over $70 \%$ lower than the loss coefficient of measured eccentric stenosis of the same degree.

Measured data show no evident difference in the severity of the $75 \%$ eccentric stenosis with different degree of eccentricity. Figure 2 plots the result of data analysis; the relationship between stenosis severity and pressure loss coefficient. These results show no important difference between loss coefficients of both eccentric stenoses but these coefficients are significantly higher than the loss coefficient of concentric stenosis.

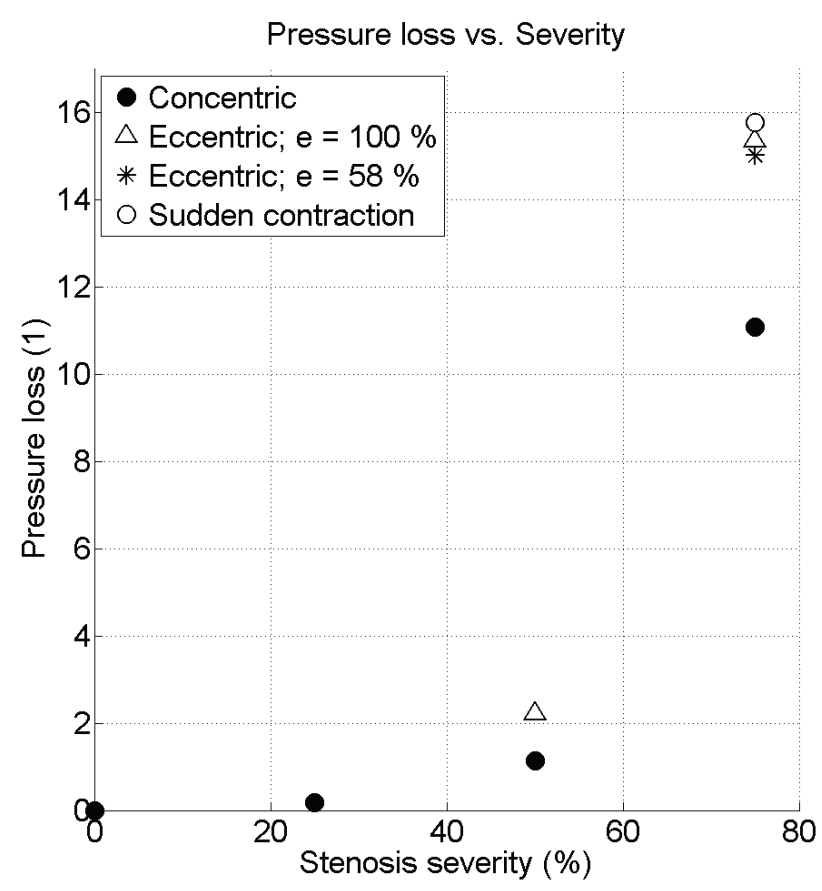

Fig. 2. Relationship between stenosis severity and loss coefficient.

The overall functional effect of all stenoses is represented by the predicted pressure drop for fixed inlet conditions (Fig. 3). The Equation 2 was utilized for pressure drop calculation for each stenosis under physiological conditions as follows: the artery with diameter of $5 \mathrm{~mm}$ and blood density of $1060 \mathrm{~kg} / \mathrm{m}^{3}$. The blood flow rate was considered from 400-2000 $\mathrm{ml} / \mathrm{min}$. Curves are plotted within the range of valid Reynolds number. Calculated pressure loss increases with stenosis severity; the pressure loss for all asymmetric stenoses is higher than for concentric one. Sudden contraction did not differ considerably.

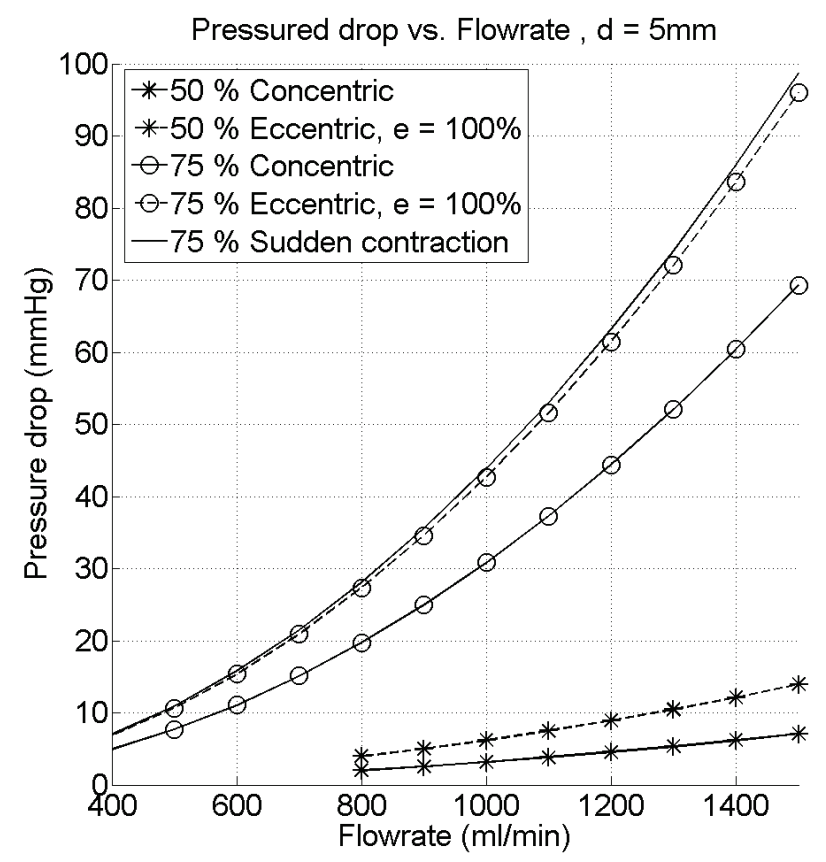

Fig. 3. Pressure drop vs. flow rate. Flow rate $\mathrm{Q}=400-2000$ $\mathrm{ml} / \mathrm{min}$, artery diameter $\mathrm{d}=5 \mathrm{~mm}$.

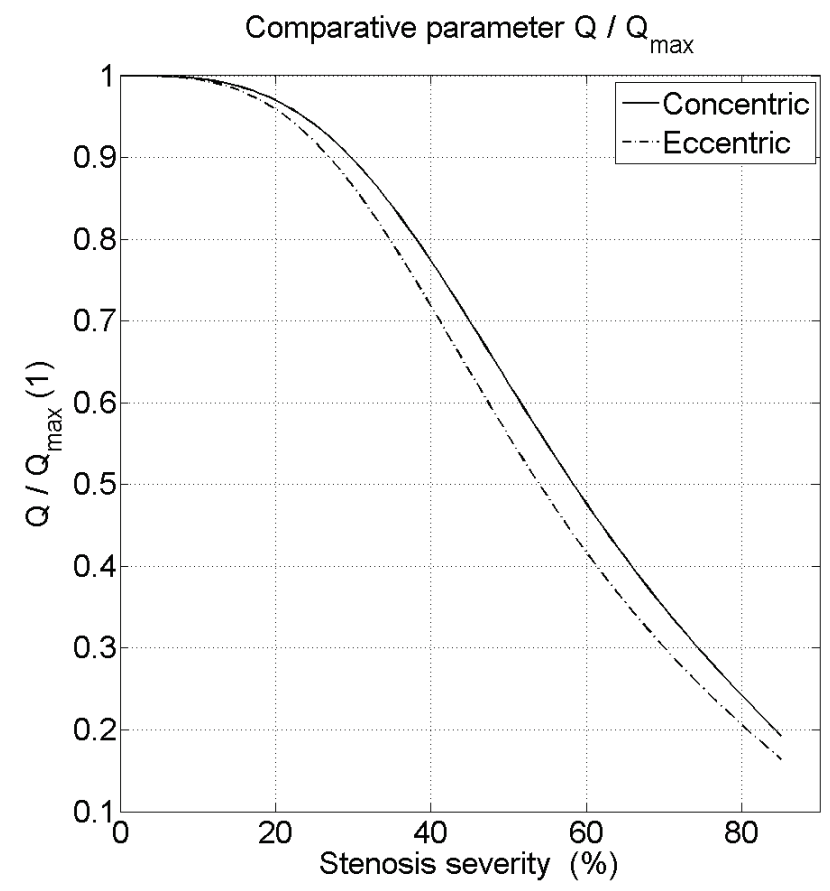

Fig. 4. Relationship between stenosis functional severity and degree of area reduction for concentric and eccentric stenosis $(e=100 \%)$. 
Relationship between $Q / Q_{\max }$ and degree of area reduction are plotted in Figure 4 to more clearly reveal the functional severity of stenosis. Smooth lines were constructed using interpolated data of loss coefficients. The flow rate does not change significantly until $20 \%$ of area reduction (Fig. 4). The effect of the stenosis measured by the $Q / Q_{\max }$ parameter increases rapidly for stenosis over $60 \%$ area reduction where the flow rate is less than $50 \%$ in comparison with the flow rate through unconstricted tube. Figure 5 shows the variation of pressure drop with eccentricity for $75 \%$ stenosis. The results exhibit increase of pressure drop for non-zero eccentricity. The conditions for calculations were: density of blood of $1060 \mathrm{~kg} / \mathrm{m}^{3}$, artery diameter of $5 \mathrm{~mm}$ and blood flow rate $\mathrm{Q}=600 \mathrm{ml} / \mathrm{min}$.

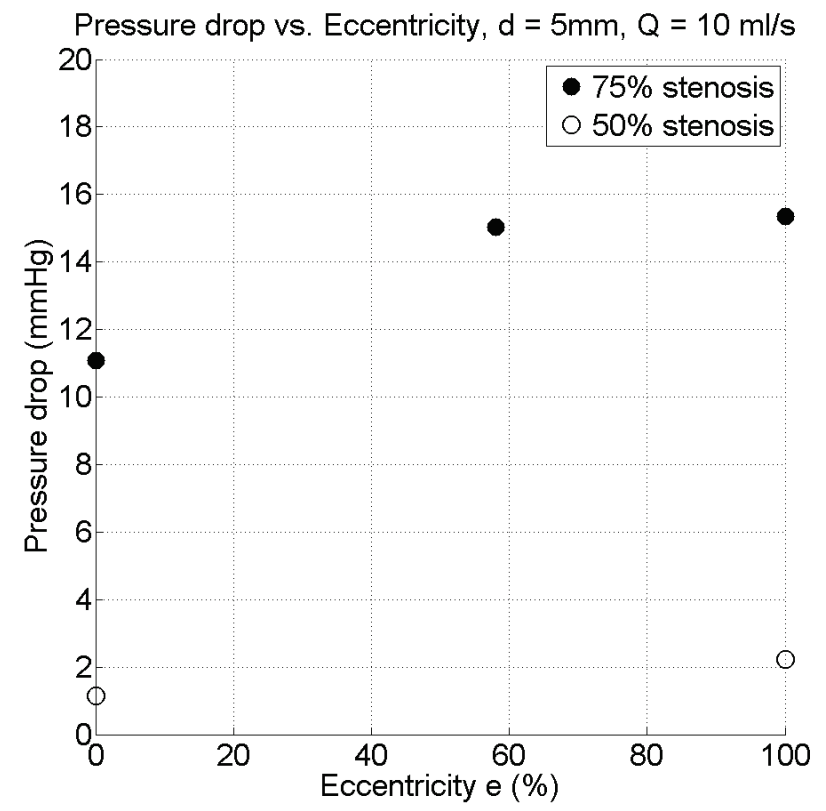

Fig. 5. Variation of pressure drop with stenosis eccentricity ratio. Flow rate $\mathrm{Q}=600 \mathrm{ml} / \mathrm{min}$, artery diameter $\mathrm{d}=5 \mathrm{~mm}$.

\section{Discussion}

This study has shown that stenosis asymmetry is associated with more profound pressure drop and flow volume decrease. On the contrary, pressure drop and flow volume decrease were not further significantly influenced by the level of asymmetry.

From all investigated geometries, the concentric stenosis induced the smallest hydrodynamic changes. This observation could be explained by the fact, that the non-symmetric geometry creates enlarged recirculation zones, distal to the stenosis. The eccentricity leads to the development of downstream circumferentially non- uniform secondary flow and increases the energy dissipation. Our results showed that the pressure drop of stenosis with $75 \%$ area reduction was increased of approximately $35 \%$ in the range of eccentricity $0-58 \%$. The higher value of eccentricity was not associated with additional pressure drop increase. As far as the level of asymmetry does not further significantly influence the hydrodynamic changes, we can postulate that the concentric geometry of the stenosis is the ideal one to ensure blood flow as high as possible. Indeed, the positive remodeling of arterial segments with atheromatous plaques tends to make the stenosis axisymmetric (Schoenhagen et al. 2001). The numerical simulation results of Heflin et al. (2009) got similar values of pressure drop. Their study indicated a critical stenosis percentage of approximately 75-80\% above which the pressure loss increases substantially, which is in good agreement with our observation, but their study did not observe a dependence of the pressure loss on eccentricity. On the contrary, experiments of Lundin et al. (2002) proved the eccentric stenosis creates higher pressure drop than concentric stenosis, but they did not measure the flow volume decrease.

The inadequacy of "stenosis percentage" alone in characterizing flow distal to the lesion has been also pointed out by Tang et al. (2013). Their study showed that eccentricity is another important parameter to describe such flows more accurately - the eccentricity caused more profound changes in wall shear stress and strain - known factors leading to the acceleration of atherogenesis and of neointimal hyperplasia in stents and dialysis accesses (Malik et al. 2014).

In our experiments the pressure drop was measured for steady flow, which is a possible limitation. Several studies have reported that the addition of oscillations to steady flow increased the flow resistance (Carpinlioglu and Gundogdu 2001, Hershey and Song 1967). However, the effect of oscillation amplitude on flow resistance is not completely clear. According to the relations published in Young et al. (1972), the increase in loss coefficient is less than $5 \%$ for pulsatile flow regimes in human body. These authors discussed later (Young et al. 1975) that unsteady flow through a stenosis produces pressure drop that is only slightly higher than the corresponding value for steady flow. The explanation is in the inertial effects of unsteady flow. The relative influence of this term decreases with the stenosis severity. Therefore, we think that our results are plausible also for the pulsatile flow in arteries. From the practical point of 
view, the use of steady flow in the in vitro experiments is less tricky and allows more precise measurements of pressure changes.

In this study pure water was used as the working fluid. Blood is a non-Newtonian liquid and does not exhibit a constant viscosity at all flow rates. The nonNewtonian behavior is most significant in small branches and capillaries. In most arteries, blood behaves in a Newtonian fashion, and the viscosity can be taken as a constant (Liou and Liou 1999, Ku 1997, Leuprecht and Perktold 2001). At the flow rates and vessel dimension used in these experiments, the effect of the nonNewtonian characteristics of blood is minimal.

Our results demonstrate that stenosis asymmetry considerably increases its hemodynamical effects, described by the more profound decrease of pressure and flow volume, in comparison to axisymmetric stenoses. Moreover, literary data show that asymmetric stenoses are also associated with larger areas of low, oscillating shear stress, leading to faster progression of the stenosis. Simple description of a given stenosis only by the percentage of area reduction is therefore inadequate. From the clinical point of view, the results of this study are important especially in case of borderline arterial or dialysis access stenoses when the intervention is considered. Stenosis asymmetry could be an additional factor in decision making.

\section{Conflict of Interest}

There is no conflict of interest.

\section{Acknowledgements}

The project was supported by the grants NT14161-3 and NT14160-3 of the Internal Grant Agency, Ministry of Health, Czech Republic.

\section{References}

CARPINLIOGLU MO, GUNDOGDU MY: A critical review on pulsatile pipe flow studies directing towards future research topics. Flow Meas Instrum 12: 163-174, 2001.

DODDS SR: The haemodynamics of asymmetric stenoses. Eur J Vasc Endovasc Surg 24: 332-337, 2002.

GROVES EM, SETO AH, KERN MJ: Invasive testing for coronary artery disease: FFR, IVUS, OCT, NIRS. Cardiol Clin 32: 405-417, 2014.

HEFLIN LA, STREET CB, PAPAVASSILIOU DV, O'REAR EA: A computational investigation of the geometric factors affecting the severity of renal arterial stenoses. J Biorheol 23: 102-110, 2009.

HERSHEY D, SONG G: Friction factors and pressure drop for sinusoidal laminar flow of water and blood in rigid tubes. AIChE J 13: 491-496, 1967.

IDELCHIK IE: Handbook of Hydraulic Resistance. 3rd edition. Jaico Publishing House, Humbai, 2005.

KU DN: Blood flow in arteries. Ann Rev Fluid Mech 29: 399-434, 1997.

LEUPRECHT A, PERKTOLD K: Computer simulation of non-Newtonian effects on blood flow in large arteries. Comput Methods Biomech Biomed Engin 4: 149-163, 2001.

LIOU TM, LIOU SN: A review on in vitro studies of hemodynamic characteristics in terminal and lateral aneurysm models. Proc Natl Sci Counc ROC(B) 23: 133-148, 1999.

LIU B: The influence of stenosis on the downstream flow pattern in curved arteries. Med Eng Phys 29: 868-876, 2007.

LUNDIN S, METCALFE RW, HARTLEY CJ: Effects of severity and eccentricity of carotid stenosis on pulsatile blood flow. Proc Joint EMBS/BMES, 1311-1312, 2003.

MALIK J, KUDLICKA J, TUKA V, CHYTILOVA E, ADAMEC J, ROCINOVA K, TESAR V: Common carotid wall shear stress and carotid atherosclerosis in end-stage renal disease patients. Physiol Res 61: 355-361, 2012.

MALIK J, KUDLICKA J, NOVAKOVA L, ADAMEC J, MALIKOVA H, KAVAN J: Surveillance of arteriovenous accesses with the use of duplex Doppler ultrasonography. J Vasc Access 15 (Suppl 7): S28-S32, 2014.

PIELHOP K, KLAAS M, SCHRÖDER W: Analysis of the unsteady flow in an elastic stenotic vessel. Eur $J$ Mech B Fluids 35: 102-110, 2012.

ROY AS, BACK LH, BANERJEE RK: Guidewire flow obstruction effect on pressure drop-flow relationship in moderate coronary artery stenosis. $J$ Biomech 39: 853-864, 2006. 
SCHOENHAGEN P, ZIADA KM, VINCE DG, NISSEN SE, TUZCU EM: Arterial remodeling and coronary artery disease: the concept of "dilated" versus "obstructive" coronary atherosclerosis. J Am Coll Cardiol 38: 297-306, 2001.

SHUIB AS, HOSKINS PR, EASSON WJ: Flow visualization and characterization of an artery model with stenosis. Eng Technol 60: 56-69, 2011.

TANG D, YANG C, KOBAYASHI S, ZHENG J, VITO RP: Effect of stenosis asymmetry on blood flow and artery compression: a three-dimensional fluid-structure interaction model. Ann Biomed Eng 31: 1182-1193, 2003.

WILMINK T: Lower limb access. J Vasc Access 15 (Suppl 7): S130-S135, 2014.

YOUNG DF, TSAI FY: Flow characteristics in models of arterial stenosis: I. Steady flow. J Biomech 6: 395-410, 1972.

YOUNG DF, TSAI FY: Flow characteristics in models of arterial stenosis: II. Unsteady flow. J Biomech 6: 547-559, 1973.

YOUNG DF, CHOLVIN NR, ROTH AC: Pressure drop across artificially induced stenoses in the femoral arteries of dogs. Circ Res 36: 735-743, 1975. 